\title{
Housing Concerns Faced by Immigrant Women Dealing with Intimate Partner Violence: Findings of a Qualitative Study in Vancouver, Canada
}

Sepali Guruge* Margareth Zanchetta and Sadaf Seifi

Daphne Cockwell School of Nursing, Ryerson University, Toronto, Canada

*Corresponding author: Sepali Guruge, Professor, Daphne Cockwell School of Nursing, Ryerson University, Toronto, Canada , Tel: 416-979-5000; E-mail: sguruge@ryerson.ca

Received date: Dec 05, 2018; Accepted date: Dec 12, 2018; Published date: Dec 19, 2018

Copyright: (c) 2018 Seifi S. This is an open-access article distributed under the terms of the Creative Commons Attribution License, which permits unrestricted use, distribution, and reproduction in any medium, provided the original author and source are credited.

\begin{abstract}
This paper presents the results of a qualitative, interpretive, descriptive, study that explored the lived experiences of immigrant women who had experienced intimate partner violence and consequently were facing housing concerns in Vancouver, Canada. The data were generated through 15 individual interviews with immigrant women $(n=11)$ who had experienced intimate partner violence, as well as service providers $(n=4)$. Analyses explored how a lack of adequate housing in the context of experiencing intimate partner violence affected the safety and stability of immigrant women. The findings revealed that some women were left on the verge of homelessness, and that housing needs are even more pronounced for immigrant women who face language barriers and tend to lack financial means and established support systems.
\end{abstract}

Keywords: Canada; Housing; Immigrant women; Immigration status; Intimate partner violence; Vancouver

\section{Literature Review}

In Canada, immigrants tend to settle in urban areas that appear economically prosperous, believing they will secure a successful living situation. However, newcomers often find that the reality of life in urban settings is overwhelming, especially in terms of housing security. The ability to secure housing lies on a continuum and can be affected by factors such as health, gender, and racialized status [1,2]. Housing stability is known to benefit the pursuit of education, as well as the ability to find and maintain employment, and to help individuals recover from trauma. In contrast, a lack of stable housing has been linked with higher rates of unemployment, increased stress, and a longer and more challenging process of settlement and integration among immigrants $[1,3]$.

More than $60 \%$ of all immigrants to Canada (regardless of length of residence in the country) and more than $70 \%$ of newcomers (the first few years) move to Toronto, Vancouver, or Montreal, the country's three largest metropolitan centres. Most large cities in Canada provide housing for low-income populations, including market-rent housing (rental units in designated buildings where rents are set according to local rates, subsidized housing (rent-geared-to-income where rent is about $30 \%$ of gross income), and supportive housing (rental subsidies along with ongoing targeted support services for residents). The Metro Vancouver housing market is among the most expensive in Canada, and many individuals, including Canadian-born individuals and families, find it difficult to secure adequate and affordable housing in the city. Research has revealed that the street and homeless populations in Vancouver include disproportionate numbers of racialized individuals [4,5]. Immigrants face additional barriers to settlement, including unfamiliar social and political practices related to education, jobs, housing, etc. Some immigrants who face housing insecurity are referred to as the 'hidden' homeless because they may be living in illegal or temporary accommodations [4,5].
Immigrant women are known to face additional barriers related to immigrant status, racialized status and culture, and housing instability is one more impediment to settlement. Immigrant women who are dealing with intimate partner violence (IPV) are extremely vulnerable and often live in conditions of poverty, housing instability, danger, and exploitation [6]. The few Canadian studies that have explored the link between IPV and housing insecurity have found that IPV is the most common reason why immigrant women leave a stable housing situation [7]. Thurston et al.s multi-site study carried out in Calgary, Winnipeg, and Halifax found that housing insecurity, in turn, forces women to return to their abuser [7].

In Canada, family shelters were initially intended as a resource in situations of crisis, but many are now functioning as transitional and supportive housing for women who have experienced IPV, along with their children [8]. Paradis et al. found that rather than having to live in overcrowded conditions in shelters with people whose situations differ greatly from their own, transitional and supportive housing serves as a more 'home-like' environment where women and children can stabilize their lives and access appropriate services [6]. Immigrant women facing IPV are in greater need of alternative housing because they may not have any established support networks that could provide temporary accommodation.

\section{Methods}

The purpose of our study was to explore the housing needs and experiences of immigrant women with varying degrees of immigration status when dealing with IPV in the post-migration context in Canada. We focused on individual narratives shared by immigrant women, as well as the concerns and actions of frontline service providers. Interviews explored issues pertaining to perceptions about and availability of housing options, influence of gender and culture, and the role of service providers and advocates.

This study applied an interpretive, descriptive design, which allows for flexible inquiry into the particular experiences of individuals and 
how they choose to communicate these experiences in unique ways $[9,10]$.

Participants included immigrant women who were experiencing or had experienced IPV, as well as service providers who work with women experiencing IPV. Inclusion criteria for women were: immigrant woman (regardless of immigration status in Canada); able to communicate in English; at least 18 years of age; experience of IPV; and looking or looked for housing in Metro Vancouver. Inclusion criteria for service providers were: professional experience working with immigrant women who were dealing with IPV and housing in Metro Vancouver.

Potential participants were recruited through community agencies and non-profit organizations working with immigrant women. They were approached by phone or email, and then initial participants helped find additional participants (snowball sampling). All potential participants were informed about the study via telephone, and were asked to read and sign a consent form before being interviewed. They were informed about their right to refuse participation or to refuse to answer any questions, to stop the interview at any time, and to withdraw from the study at any time without giving reasons or affecting their future care/services. The study protocol was approved by University's Research Ethics Board.

Interviews with immigrant women took place in a private room at an immigrant-serving agency that was easily accessible to participants and provided a safe environment. Each semi-structured interview lasted approximately one hour and included questions about reasons for leaving their home country, experiences within their relationships and how violence emerged, factors that prevented them from leaving an abusive relationship, access to housing, and experiences related to settlement in Metro Vancouver. Interviews were audio-recorded with the consent of participants. Each participant received a small honorarium (\$30) to cover the costs of childcare, transportation, and their time.

The first stage of data analysis involved data preparation and organization [11]. Audio recordings of interviews were transcribed by the first author, along with any notes taken during interviews. Next, data were analyzed using inductive thematic analysis, enabling the identification of patterns and important themes, as well as contrasts and comparisons across data from individual participants and datasets [12]. Data-coding and final interpretations of the findings were reviewed and confirmed by the second author.

\section{Results}

We conducted 15 interviews: 11 with immigrant women and 4 with service providers. Immigrant women were from Iran, Japan, Korea, Mexico, Pakistan, Romania, Sudan, and Turkmenistan. At time of interview, 2 were Canadian citizens, 6 were permanent residents, and 3 reported having no legal status. Their ages ranged from 25-40 years, and all of them were mothers who had separated or divorced after having experienced IPV. The 4 interviews with service providers working with immigrant women in the Metro Vancouver Region included: a multicultural outreach worker, a legal advocate, a transition house coordinator, and a Stopping the Violence counselor. They were all women, aged $30-45$ years.

The next section presents the key themes that emerged from the data along with excerpts.

\section{Lack of affordable housing being the biggest college}

Interviews revealed that it was more difficult for immigrant women who did not belong to the economic or business class to find affordable housing, compared with economic or business class immigrant women. Interviewees reported a range of barriers to housing related to affordability: low income in relation to high rent, long waitlists for social housing, and a lack of resources to accommodate more than one or two children. Housing options were affected by women's financial situations and the availability of subsidized housing:

It's a question of how do I make sure that I've got my rent or food on the table? (Permanent Resident, from Sudan)

Affordability was an issue for the majority of participants. Many were seeking affordable housing and others were readjusting after having had to live with other families or roommates. The search for housing, along with other pressures such as job insecurity and ongoing IPV, had substantial negative effects on their ability to settle in Canada:

I was with my son; if I was alone I could have slept on the street it would have been okay but with my son, I didn't know what I could do. My biggest concern was my son, and the environment's effects on him psychologically. (Permanent Resident, from Korea)

Most victims of abuse had no family in Canada to rely on for support. They said that Vancouver has long affordable housing waitlists for social, subsidized, and transitional housing. One of the service providers confirmed these challenges in the housing market:

We always help them register for BC Housing but that is normally a two year wait so for more volatile situations, this is not an option. (Multicultural Worker)

In addition to long waitlists, many housing units that the women visited were in poor condition with bad smells and dirty carpets, and were located in unsafe neighborhoods. One interviewee acknowledged that although she was not in a position to be picky, she rejected many of the available options due to their poor upkeep and undesirable conditions. Another described her current living conditions in a subsidized suite provided by BC Housing:

There is a lot of damage in the house... the cupboard even fell on my face and injured me. I told the manager ... he said...it was my fault... I have to pay money to fix it. It is not safe for me ... for my little kids. (Permanent Resident, from Sudan)

Income was a huge barrier to housing security. The majority of women were dependent on some type of income support. For example, government assistance provided the women with some independence, but this income was inadequate to support adequate housing and other basic needs. Additionally, women who did not have legal status were not eligible for income assistance; one commented:

People... have welfare and can get approved by BC Housing and move into subsidized housing places but I had no way to get into them... I got $\$ 580$ from spousal support...paid $\$ 400$ for my place... and... \$100 per month for myself and children [over] the last 3 years... (No Legal Status, from Japan)

In many cases, a lack of information about where to get help or how to find accommodation forced women to spend several nights at the airport, shelters, or the homes of friends or acquaintances. These difficulties were compounded by gendered cultural factors, such as shame and stigma about leaving the husband, and/or living on their own, as well as becoming the head of the family for the first time. 
Interviewees stressed the importance of providing new immigrants with information about a number of topics, including services related to housing options, particularly for women in emergency situations.

One service provider referred to the concept of a 'false choice' with regard to housing, citing comments made by a local city councillor about how affordable housing in Vancouver means that 'someone' would be able to afford it, implying that different people prioritize different things.

... These Housing First models are showing, that if you have housing, you can fix other problems...if you don't get housing until you fix the other problems in your life then people don't fix these problems... we are setting people up so that that way they lose their housing... (Legal Advocate)

This service provider felt this is a problematic way of thinking that "everyone prioritizes having a roof over their head, but not everyone can afford to spend three-quarters of their income on housing."

\section{Transition homes as a critical stepping stone}

Some interviewees said they were unaware of the existence of transition homes before the crisis that forced them into an emergency shelters or first-stage transition home. Others spoke of their worries about transition homes, which stemmed from their own assumptions and misconceptions, as well as comments from friends and acquaintances. One woman described her preconceived notion regarding transition homes:

I thought people are there because of alcohol abuse, smoking, drugs...but when I went it was families, in the same situation as me. (No Legal Status, from Mexico)

This type of negative image of what it would be like to live in a transition home was common. The immigrant women participants felt that they would be judged for being residents of a transition house. One woman explained that this was hurtful, but that at the same time she knew that she and her son were lucky to be there, safe from violence. Another interviewee commented:

I applied to second stage...I had heard of the reputation here [Surrey] so I was a bit concerned but when I came for my interview, it was a really good surprise. It was really a clean and beautiful building, everything was provided... (Canadian Citizen, from Pakistan)

Although the women living in transition homes were grateful to be there, they were aware that it was only temporary. As a result, most were seeking affordable housing to move into before their time at the transition home ended. However, long waitlists for subsidized housing jeopardized the feeling of safety they had recently regained after settling into the transition homes.

One service provider noted that it is even more difficult for those who have not stayed in transition homes to gain quick access to subsidized housing. She explained that it is extremely difficult to get her outreach clients into affordable housing, because they tend not to have anyone directly responsible for their file. In contrast, after serving as a transition house landlord, she was able to support the applications of her transition house tenants and act as a reference. All four service providers referred to similar difficulties in assisting women who were leaving abusive partners with their housing situations.

\section{The critical role of service providers}

The majority of the immigrant women interviewed said that they were only able to find affordable housing through the advocacy and persistence of service providers. One said:

I was supposed to have a steady income to get in here but I had no status but the worker said she could help me get income assistance for hardship and once I get that then I could have a steady income and become a resident here... (No Legal Status, from Japan)

Women who had not spent any time at a transition home and were not in contact with service providers had extreme difficulty even filling out the application to get onto a waitlist. The service providers agreed that the application process in itself is a barrier, even for Canadianborn women. Immigrant women reported that having someone assist with the lengthy application process helped relieve their stress. Service providers helped clients with a wide array of supports, such as accompanying them to appointments or court hearings and writing letters of support for housing, income assistance, and/or childcare subsidies. While long wait times are common in all forms of subsidized housing provided by BC Housing, service providers ensured that women escaping IPV were placed on priority lists. Their wellestablished networks within and outside of their own areas of expertise were extremely helpful in assisting women to secure housing, even if they lacked legal status:

I stayed in the first stage transition home for 30 days... but with the help of my case worker for violence and abuse, they were able to extend my stay until they helped me find a more permanent place to live. (No Legal Status, from Turkmenistan)

One service provider explained:

[They] work really hard to get the mothers without status into permanent housing. [The service providers] make sure that they [the mothers] are on income assistance. Often just receiving child support seems to be sticky... [they] work hard to build strong enough relationships that [they] can vouch for these women. (Coordinator of a Transition Home)

Along with supporting women through the trauma of IPV, aiding them in their search for housing, and assisting them with income and other subsidies, all four service providers emphasized the need to empower their clients and to advocate for them. They had a common mission of empowering women to be independent and lead lives free of violence and abuse. One counsellor said that they "want to support women to make their own decisions." Service providers supported women in whatever choices they made and helped ensure they had all appropriate resources. However, the lack of available funding or resources frequently made it difficult or impossible to help.

\section{Discussion}

All of the immigrant women who had experienced IPV had also experienced critical housing stress. This is the case for most marginalized and low-income populations in Vancouver, but our study participants faced unique challenges related to their experiences of IPV, uncertain immigration status, racialized status, lack of social networks, and language barriers. As long as housing is considered a privilege rather than a right, women and children in similar situations will continue to experience the same problems. Government policies dictate whether women and children fleeing abuse will be homeless or not: they are often unable to find housing that is both secure and 
affordable; the scarce supply of affordable housing results in "high rent-to-income ratios among the least well off and puts them at-risk for economically-induced homelessness" [4].

When women and their children fleeing abuse can find affordable housing, they can begin to re-establish their lives. The service providers we interviewed confirmed that once their clients find secure and affordable housing, they feel more empowered to deal with other challenges they and their children face. Programs aimed at strengthening individual resilience are also important, but without housing, women are unable to focus on other important issues such as education, employment, health, and improving the lives of their children.

Funding is another systemic barrier to providing services. Service providers and clients expressed frustration about being limited to a certain amount of outreach hours. Additionally, the subsidies granted to women after they leave an abusive spouse are extremely small, and this is compounded by the fact that they may lack language abilities and/or access to childcare that would enable them to find employment. An increase in income assistance could help the women find suitable housing so they can focus on addressing these issues.

It is also important to note that when a woman is receiving income assistance, there is a certain threshold that cannot be surpassed in terms of earned income. If a victim does find employment, her income assistance may be cut. This is another form of control that the woman has to live with, forcing her to continue to live in difficult and impoverished conditions.

\section{Limitations}

This is one of the first studies in Vancouver, one of the key immigrant-receiving metropolises in Canada, to examine the intersection of IPV, immigrant status, and housing. The findings could be strengthened by including a larger study sample to help capture similarities and difference across diverse immigrant statuses, ages, languages, lengths of stay in Canada, family size, as well as immigrantreceiving settings, such as Toronto and Montreal.

\section{Implications for practice and policy}

The study findings have some implications for practice and policy. More funding and services are needed for immigrants, especially those who are escaping IPV. For example, more funding should be available for social assistance subsidies. Housing allocation policies should be updated to increase safe and affordable housing and address the long waitlists for all types of subsidized housing. More coordination and integration among inter-sectorial agencies would promote better understanding of the issues facing immigrant women experiencing IPV and housing insecurity among policymakers, decision-makers, managers, and practitioners.

Immigration falls under federal jurisdiction, but local and provincial governments are responsible for services related to justice and equity. When immigrant women form a large proportion of the populations in shelters, transition homes, or subsidized housing units, more federal assistance is needed to assist local and provincial governments in providing access to housing for this population.

The findings also have implications for educational policy. For example, immigrants, especially women, need information about available programs and services, equality, rights, immigration policies, and laws. They should be offered functional education to help them complete various forms and applications (e.g., income assistance, immigration applications, housing applications, etc.). They should also be provided with information about their rights related to immigration status and rules regarding sponsorship breakdowns. Education will also help prevent common misconceptions among immigrant women, such as the nature of transition housing.

Finally, public awareness campaigns about IPV could support collective actions to encourage the empowerment of women and address inequities in housing markets and the various options women and their children have in terms of transition homes, subsidized housing, and the rental market.

\section{Conclusions}

Together, our findings revealed that the process of finding safe and affordable housing is not a simple matter of finding shelter - it is about finding resources that can support the complex and multiple contexts and needs these women are facing. Secure, safe, and stable housing for all women and children, including immigrant women escaping IPV and their children, should be a priority for policymakers, and systemic barriers must be addressed. More research is also needed to include women of diverse immigration statuses, ages, education levels, racialized statuses, lengths of stay in Canada, and health conditions, which could all influence vulnerability and resilience.

\section{Acknowledgements}

We sincerely thank the women who shared their stories with us, and for allowing us to share them on behalf of survivors of IPV who are struggling to find housing. We also thank the service providers who participated in the study and those who helped us during the recruitment phase.

\section{References}

1. Francis J, Hiebert D (2014) Shaky foundations: Refugees in Vancouver's housing market. The Canadian Geographer 58: 63-78.

2. Thurston W, Clow B, Este D, Gordey T, Haworth-Brockman M, et al. (2006) Immigrant women, family violence, and pathways out of homelessness. Report for the National Secretariat on Homelessness.

3. Gillard G (2014) Fostering inclusion for newcomers to Ottawa: A CHRA session case study. The Canadian Housing and Renewal Association. Ottawa, ON.

4. Fiedler R, Schuurman N, Hyndman J (2006) Hidden homelessness: An indicator-based approach for examining the geographies of recent immigrants at-risk of homelessness in Greater Vancouver. Cities 23: 205-216.

5. Hiebert D, Germain A, Murdie R, Preston V, Renaud J, et al. (2006) The housing situation and needs of recent immigrants in the Montréal, Toronto, and Vancouver CMAs: An overview. Canada Mortgage and Housing Corporation.

6. Paradis E, Novak S, Sarty M, Hulchanski JD (2008) Better off in a shelter? A year of homelessness \& housing among status immigrant, non-status migrant \& Canadian-born families. Centre for Urban and Community Studies Cities Centre, University of Toronto.

7. Thurston W, Roy A, Clow B, Este D, Gordey T, et al. (2013) Pathways Into and Out of Homelessness: Domestic violence and housing security for immigrant women. Journal of Immigrant and Refugee Studies 11: 278-298.

8. Kaur H, Porteous T, Kamaljit KL, Ruebsaat G, Bassi B, et al. (2012) Immigrant Women's Project: Safety of immigrant, refugee and non-status women. Ending Violence Association of BC, MOSAIC \& Vancouver \& Lower Mainland Multicultural Family Support Services Society. 
Citation: Guruge S, Zanchetta M, Seifi S (2018) Housing Concerns Faced by Immigrant Women Dealing with Intimate Partner Violence: Findings of a Qualitative Study in Vancouver, Canada. Arts Social Sci J 9: 427. doi:10.4172/2151-6200.1000427

Page 5 of 5

9. Thorne S, Reimer-Kirkham S, O’Flynn-Magee K (2004) The analytic challenge in interpretive description. International Journal of Qualitative Methods 3: 1-21.

10. Elliott R, Timulak L (2005) Descriptive and interpretive approaches to qualitative research. In P Gilbert \& J Miles (Editors), A handbook of research methods for clinical and health psychology. Oxford University Press: 147-159.
11. Creswell JW, Hanson WE, Clark Plano VL, Morales A (2007) Qualitative research designs: Selection and implementation. The counseling psychologist 35: 236-264.

12. Bryman A (2001) Social Research Methods. Oxford: Oxford University Press. 